\title{
Observational constraints on holographic dark energy with varying gravitational constant
}

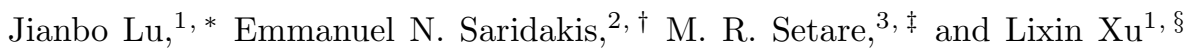 \\ ${ }^{1}$ Institute of Theoretical Physics, School of Physics 83 Optoelectronic Technology, \\ Dalian University of Technology, Dalian, 116024, P. R. China \\ ${ }^{2}$ College of Mathematics and Physics, \\ Chongqing University of Posts and Telecommunications, Chongqing, 400065, P.R. China \\ ${ }^{3}$ Department of Science, Payame Noor University, Bijar, Iran
}

\begin{abstract}
We use observational data from Type Ia Supernovae (SN), Baryon Acoustic Oscillations (BAO), Cosmic Microwave Background (CMB) and observational Hubble data (OHD), and the Markov Chain Monte Carlo (MCMC) method, to constrain the cosmological scenario of holographic dark energy with varying gravitational constant. We consider both flat and non-flat background geometry, and we present the corresponding constraints and contour-plots of the model parameters. We conclude that the scenario is compatible with observations. In $1 \sigma$ we find $\Omega_{\Lambda 0}=0.72_{-0.03}^{+0.03}, \Omega_{k 0}=$ $-0.0013_{-0.0040}^{+0.0130}, c=0.80_{-0.14}^{+0.19}$ and $\Delta_{G} \equiv G^{\prime} / G=-0.0025_{-0.0050}^{+0.0080}$, while for the present value of the dark energy equation-of-state parameter we obtain $w_{0}=-1.04_{-0.20}^{+0.15}$.

PACS numbers: 95.36.+x, 98.80.-k, 98.80.Es
\end{abstract}

\section{INTRODUCTION}

Nowadays it is strongly believed that the universe is experiencing an accelerated expansion, and this is supported by many cosmological observations, such as SNe Ia [1], WMAP [2], SDSS [3] and X-ray [4]. A first direction that could provide an explanation of this remarkable phenomenon is to introduce the concept of dark energy, with the most obvious theoretical candidate being the cosmological constant. However, at least in an effective level, the dynamical nature of dark energy can also originate from a variable cosmological "constant" [5], or form various fields, such is a canonical scalar field (quintessence) [6], a phantom field, that is a scalar field with a negative sign of the kinetic term [7], or the combination of quintessence and phantom in a unified model named quintom [8]. The second direction that could explain the acceleration is to modify the gravitational theory itself, such is the generalization to $f(R)$-gravity [9]), scalar-tensor theories with non-minimal coupling [10], string-inspired models [11] etc.

Going beyond the aforementioned effective description requires a deeper understanding of the underlying theory of quantum gravity unknown at present. However, physicists can still make some attempts to probe the nature of dark energy according to some basic quantum gravitational principles. Currently, an interesting such an attempt is the so-called "Holographic Dark Energy" proposal [12, 13]. Its framework is the black hole thermodynamics and the connection (known from AdS/CFT correspondence) of the UV cut-of of a quantum field theory, which gives rise to the vacuum energy, with the largest distance of the theory [14]. Thus, determining an appropriate quantity $L$ to serve as an IR cut-off, imposing the constraint that the total vacuum energy in the corresponding maximum volume must not be greater than the mass of a black hole of the same size, and saturating the inequality, one identifies the acquired vacuum energy as holographic dark energy:

$$
\rho_{\Lambda}=\frac{3 c^{2}}{8 \pi G L^{2}},
$$

with $G$ the Newton's gravitational constant and $c$ a constant. The holographic dark energy scenario has been tested and constrained by various astronomical observations [15] and it has been extended to various frameworks [16 18].

However, there are indications that Newton's "constant" $G$ can by varying, being a function of time or equivalently of the scale factor [19]. In particular, observations of Hulse-Taylor binary pulsar [20, 21], helio-seismological data [22], Type Ia supernova observations [1] and astereoseismological data from the pulsating white dwarf star G117-B15A [29]

\footnotetext{
*Electronic address: lvjianbo819@163.com

†Electronic address: msaridak@phys.uoa.gr

‡Electronic address: rezakord@ipm.ir

$\S$ Electronic address: lxxu@dlut.edu.cn
} 
lead to $|\dot{G} / G| \lesssim 4.10 \times 10^{-11} y r^{-1}$, for $z \lesssim 3.5[23]$. Thus, in our previous paper [24], we investigated the holographic dark energy scenario under a varying gravitational constant, and we extracted the corresponding corrections to the dark energy equation-of-state parameter.

In the present work we are interested in constraining the model of holographic dark energy with a varying Newton's constant, using various observational data arising from SN, BAO, CMB and OHD. Such an analysis is crucial for the validity of the aforementioned scenario. The plan of the work is as follows: In section [II we present briefly the holographic dark energy scenario with a varying Newton's constant and the corresponding expressions of the darkenergy equation-of-state parameter. In section \س we perform a combined observational constraint analysis, allowing for variations in all model parameters. Finally, section $[\mathrm{IV}$ is devoted to the summary of our results.

\section{HOLOGRAPHIC DARK ENERGY WITH VARYING GRAVITATIONAL CONSTANT}

In this section we briefly review the holographic dark energy proposal, in the case where the gravitational constant is itself a function of the scale factor 24]. In order for our results to be more transparent we examine separately the flat and non-flat background geometries.

\section{A. Flat universe}

In the case where the space-time geometry is a flat Robertson-Walker:

$$
d s^{2}=-d t^{2}+a(t)^{2}\left(d r^{2}+r^{2} d \Omega^{2}\right),
$$

with $a(t)$ the scale factor and $t$ the comoving time. As usual, the first Friedmann equation reads:

$$
H^{2}=\frac{8 \pi G}{3}\left(\rho_{m}+\rho_{\Lambda}\right)
$$

with $H$ the Hubble parameter, $\rho_{m}=\frac{\rho_{m 0}}{a^{3}}$, where $\rho_{m}$ and $\rho_{\Lambda}$ stand respectively for matter and dark energy densities and the index 0 marks the present value of a quantity. Furthermore, we will use the density parameter $\Omega_{\Lambda} \equiv \frac{8 \pi G}{3 H^{2}} \rho_{\Lambda}$, which, imposing explicitly the holographic nature of dark energy according to relation (11), becomes

$$
\Omega_{\Lambda}=\frac{c^{2}}{H^{2} L^{2}}
$$

As usual, in the case of a flat universe, the best choice for the definition of $L$ is to identify it with the future event horizon [12, 13, 25], that is $L \equiv R_{h}(a)$ with

$$
R_{h}(a)=a \int_{t}^{\infty} \frac{d t^{\prime}}{a\left(t^{\prime}\right)}=a \int_{a}^{\infty} \frac{d a^{\prime}}{H a^{\prime 2}} .
$$

In this case, using (4) and the Friedmann equation (3), one can show that

$$
\Omega_{\Lambda}^{\prime}=\Omega_{\Lambda}\left(1-\Omega_{\Lambda}\right)\left[1+\frac{2 \sqrt{\Omega_{\Lambda}}}{c}\right]-\Omega_{\Lambda}\left(1-\Omega_{\Lambda}\right) \Delta_{G},
$$

where primes denote the derivatives with respect to $\ln a$. In this expression the first term is the usual holographic dark energy differential equation [13], while the second term is the correction arising from the varying nature of $G$, which is quantified by the parameter $\Delta_{G} \equiv G^{\prime} / G$. Finally, concerning the dark-energy equation-of-state parameter $w$, considered as a function of the redshift $z$, we use the approximation [26]

$$
w(z) \approx w_{0}+w_{1}\left(\frac{z}{1+z}\right)
$$

with [24]

$$
\begin{aligned}
& w_{0}=-\frac{1}{3}-\frac{2}{3 c} \sqrt{\Omega_{\Lambda 0}}+\frac{\Delta_{G}}{3} \\
& w_{1}=\frac{1}{6 c} \sqrt{\Omega_{\Lambda 0}}\left(1-\Omega_{\Lambda 0}\right)\left(1+\frac{2}{c} \sqrt{\Omega_{\Lambda 0}}\right)-\frac{\left(1-\Omega_{\Lambda 0}\right) \sqrt{\Omega_{\Lambda 0}}}{6 c} \Delta_{G} .
\end{aligned}
$$


In these expressions, the index 0 stands for the value of a quantity at present, where the Hubble parameter is $H_{0}$ and the scale factor is $a_{0}=1$.

In summary, using the results of this subsection, and the usual relations $\Omega_{m}=\Omega_{m 0} a^{-3}$ (with $\Omega_{m} \equiv \frac{8 \pi G}{3 H^{2}} \rho_{m}$ the matter density parameter) and $\Omega_{\Lambda}=\Omega_{\Lambda 0} a^{-3(1+w)}$, we can write the Friedmann equation (3) in a form suitable for observational elaboration as:

$$
H^{2}=H_{0}^{2}\left\{\Omega_{m 0}(1+z)^{3}+\Omega_{\Lambda 0}(1+z)^{3[1+w(z)]}\right\}
$$

\section{B. Non-flat universe}

Let us now generalize the above result in the case of a general FRW universe with line element

$$
d s^{2}=-d t^{2}+a^{2}(t)\left(\frac{d r^{2}}{1-k r^{2}}+r^{2} d \Omega^{2}\right)
$$

in comoving coordinates $(t, r, \theta, \varphi)$, where $k$ denotes the spacial curvature with $k=-1,0,1$ corresponding to open, flat and closed universe respectively. The first Friedmann equation writes:

$$
H^{2}+\frac{k}{a^{2}}=\frac{8 \pi G}{3}\left(\rho_{m}+\rho_{\Lambda}\right)
$$

In this case, the cosmological length $L$ in (4) is considered to be [16]:

$$
L \equiv \frac{a(t)}{\sqrt{|k|}} \operatorname{sinn}\left(\frac{\sqrt{|k|} R_{h}}{a(t)}\right)
$$

where

$$
\frac{1}{\sqrt{|k|}} \operatorname{sinn}(\sqrt{|k|} y)= \begin{cases}\sin y & k=+1 \\ y & k=0 \\ \sinh y & k=-1\end{cases}
$$

A straightforward calculation using (41) and (12) leads to [24]:

$$
\Omega_{\Lambda}^{\prime}=\Omega_{\Lambda}\left[1-\Omega_{k}-\Omega_{\Lambda}+\frac{2 \sqrt{\Omega_{\Lambda}}}{c} \operatorname{cosn}\left(\frac{\sqrt{|k|} R_{h}}{a}\right)\left(1-\Omega_{\Lambda}\right)\right]-\Omega_{\Lambda}\left(1-\Omega_{k}-\Omega_{\Lambda}\right) \frac{G^{\prime}}{G}
$$

where

$$
\operatorname{cosn}(\sqrt{|k|} y)= \begin{cases}\cos y & k=+1 \\ 1 & k=0 \\ \cosh y & k=-1\end{cases}
$$

In expression (15) we have also introduced the curvature density parameter $\Omega_{k} \equiv-\frac{k}{(a H)^{2}}$. We mention that since in this work we focus on observational constraints, we have adopted the minus-sign convention for $\Omega_{k}$-definition, which is the usual one in observational works. Thus, in (15) $\Omega_{k}$ has the opposite sign comparing to [24]. Clearly, for $k=0$ (and thus $\Omega_{k}=0$ ) it results to (6). Finally, concerning the dark-energy equation-of-state parameter $w(z)$ we acquire $w(z) \approx w_{0}+w_{1}\left(\frac{z}{1+z}\right)$, with $[24]$

$$
\begin{aligned}
& w_{0}=-\frac{1}{3}-\frac{2}{3 c} \sqrt{\Omega_{\Lambda 0}+c^{2} \Omega_{k 0}}+\frac{\Delta_{G}}{3} \\
& w_{1}=-\frac{\Omega_{k 0}}{3}+\frac{1}{6 c} \sqrt{\Omega_{\Lambda 0}+c^{2} \Omega_{k 0}}\left[1-\Omega_{k 0}-\Omega_{\Lambda 0}+\frac{2}{c}\left(1-\Omega_{\Lambda 0}\right) \sqrt{\Omega_{\Lambda 0}+c^{2} \Omega_{k 0}}\right]-\frac{1}{6 c} \sqrt{\Omega_{\Lambda 0}+c^{2} \Omega_{k 0}}\left(1-\Omega_{k 0}-\Omega_{\Lambda 0}\right) \Delta_{G} .(18
\end{aligned}
$$

In summary, using the results of this subsection, we can write the Friedmann equation (12) in a form suitable for observational elaboration as:

$$
H^{2}=H_{0}^{2}\left\{\Omega_{k 0}(1+z)^{2}+\Omega_{m 0}(1+z)^{3}+\Omega_{\Lambda 0}(1+z)^{3[1+w(z)]}\right\} .
$$




\section{OBSERVATIONAL CONSTRAINTS}

Let us now proceed to a combined observational constraint analysis of the scenario at hand, allowing for variations in all the aforementioned model parameters. We use observational data from Type Ia Supernovae (SN), Baryon Acoustic Oscillations (BAO), Cosmic Microwave Background $\left[l_{A}\left(z_{*}\right), R\left(z_{*}\right), z_{*}\right]$ (CMB) and Observational Hubble Data (OHD). The precise methods are summarized in the Appendix.

In our calculations we take the total likelihood $L \propto e^{-\chi^{2} / 2}$ to be the product of the separate likelihoods of SN, $\mathrm{BAO}, \mathrm{CMB}$ and OHD. Thus, the $\chi^{2}$ is

$$
\chi^{2}\left(p_{s}\right)=\chi_{S N}^{2}+\chi_{B A O}^{2}+\chi_{C M B}^{2}+\chi_{O H D}^{2},
$$

and the parameter vector reads

$$
p_{s}=\left\{\Omega_{b} h^{2}, \Omega_{c} h^{2}, \Omega_{k}, c, \Delta_{G}\right\}
$$

In addition, we obtain the three derived parameters $\Omega_{\Lambda 0}, \Omega_{m 0}=\Omega_{b}+\Omega_{c}$ and the Hubble constant $H_{0}$, based on the above basic cosmological parameters. In our analysis, we perform a global fitting on determining the cosmological parameters using the Markov Chain Monte Carlo (MCMC) method. The MCMC method is based on the publicly available CosmoMC package [27], which has been modified to include the codes about $\mathrm{BAO}, \mathrm{CMB}\left[l_{A}\left(z_{*}\right), R\left(z_{*}\right), z_{*}\right]$ and OHD. Finally, apart from these and the two independent model parameters $c$ and $\Delta_{G}$, the basic cosmological parameters are taken in the following priors: the present physical baryon density $\Omega_{b} h^{2} \in[0.005,0.9]$, the present physical cold dark matter energy density $\Omega_{c} h^{2} \in[0.01,0.99]$, and for the non-flat case, the additional parameter $\Omega_{k} \in[-0.1,0.1]$.

Using these techniques we are able to impose constraints on the various parameters of the scenario of holographic dark energy with varying gravitational constant.

\section{A. Flat universe}

For the case of flat background geometry the cosmologically interesting parameters are the dimensionless quantities $\Omega_{\Lambda 0}, c$ and $\Delta_{G} \equiv G^{\prime} / G$, while we have to take into account the additional uncertainty in $H_{0}$. The corresponding $1 D$ and $2 D$ likelihood-contours are depicted in Fig. 1. We mention that for completeness we provide these plots not only for the parameters used in the fits, as discussed above, but also for the derived ones, although some of them are related to each other.

Additionally, in order to provide the results in a more transparent way, in Table $\llbracket$ we present the $1 \sigma$ best-fit values of the, used in the fits and derived ones, model parameters.

\begin{tabular}{c|c|c|c|c}
\hline \hline$\chi_{\min }^{2}$ & $\Omega_{\Lambda 0}(1 \sigma)$ & $c(1 \sigma)$ & $\Delta_{G}(1 \sigma)$ & $H_{0}(1 \sigma)$ \\
\hline 477.5 & $0.723_{-0.030}^{+0.026}$ & $0.80_{-0.13}^{+0.16}$ & $-0.0016_{-0.0049}^{+0.0049}$ & $70.2_{-2.5}^{+2.7}$ \\
\hline
\end{tabular}

TABLE I: The minimum value of $\chi^{2}$ and the $1 \sigma$ best-fit values of the model parameters, in the flat-background case. All quantities are dimensionless, apart from $H_{0}$ which is measured in $\mathrm{km} \mathrm{s}^{-1} \mathrm{Mpc}^{-1}$.

These figures show that the scenario at hand can be compatible with observations. Furthermore, note that $\Delta_{G} \equiv$ $G^{\prime} / G$ is restricted around zero, in a region which is in agreement with independent observations and estimation of $G^{\prime} / G$. In particular, observations of Hulse-Taylor binary pulsar B1913+16 lead to the estimation $\dot{G} / G \sim 2 \pm 4 \times$ $10^{-12} y r^{-1}[20,21]$, while helio-seismological data provide the bound $-1.6 \times 10^{-12} y r^{-1}<\dot{G} / G<0$ [22]. Similarly, Type Ia supernova observations give the best upper bound of the variation of $G$ as $-10^{-11} \mathrm{yr}^{-1} \leq \frac{\dot{G}}{G}<0[28]$, while astereoseismological data from the pulsating white dwarf star G117-B15A lead to $\left|\frac{\dot{G}}{G}\right| \leq 4.10 \times 10^{-11} y^{-1}[29]$. (See also 23] for various bounds on $\dot{G} / G$.) Since the limits of $G$-variation are given for $\dot{G} / G$ in units $y r^{-1}$, and since $\dot{G} / G=H G^{\prime} / G$, we can estimate their implied $\Delta_{G}$ substituting the value of $H$ in $y r^{-1}$. Thus, inserting an average estimation for the Hubble parameter $H \approx\langle H\rangle \approx 6 \times 10^{-11} \mathrm{yr}^{-1}\left[30\right.$ ], we obtain that $0<\left|\Delta_{G}\right| \lesssim 0.08$ [24]. In summary, as we observe from Fig. 1, $\Delta_{G}$ at $2 \sigma$ is well inside these bounds, and this offers a self-consistency test for our analysis.

For completeness, we close this subsection by estimating the $1 \sigma$ bounds of the dark energy equation-of-state parameter. In particular, since we have approximated it as $w(z) \approx w_{0}+w_{1}\left(\frac{z}{1+z}\right)$, with $w_{0}$ and $w_{1}$ given by (으), (99), we 

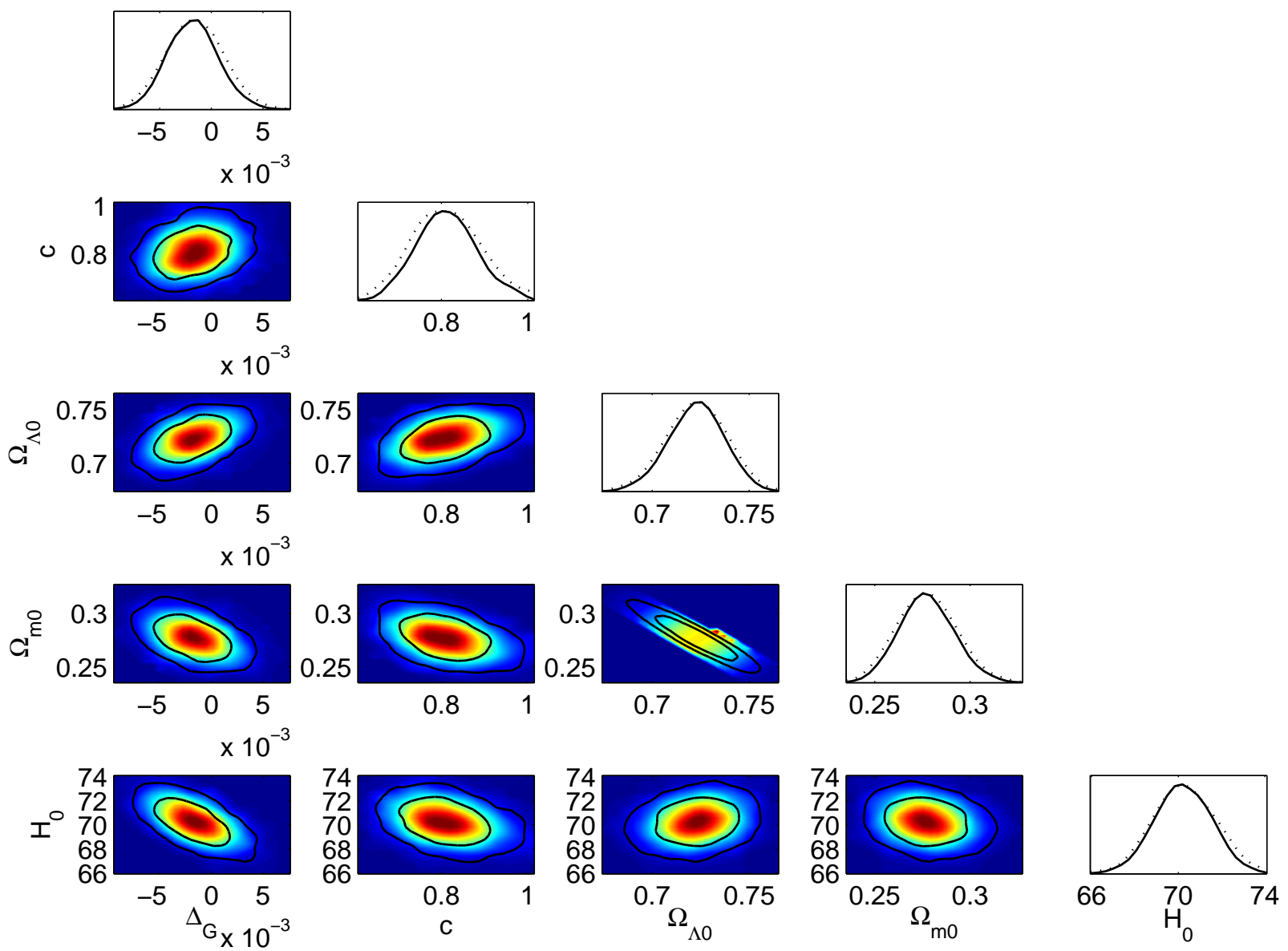

FIG. 1: (Color online) The $1 D$ and $2 D$ likelihood plots, in the flat-background case, using SN, BAO, CMB and OHD observational data. The curves stand for the $1 \sigma$ and $2 \sigma$ regions. All quantities are dimensionless, apart from $H_{0}$ which is measured in $\mathrm{km} \mathrm{s}^{-1} \mathrm{Mpc}^{-1}$.

can easily calculate that in $1 \sigma$ :

$$
\begin{aligned}
& w_{0}=-1.04_{-0.12}^{+0.13} \\
& w_{1}=0.15_{-0.03}^{+0.04} .
\end{aligned}
$$

Interestingly enough, we observe that in this scenario the best-fit value of the present value of $w$, namely $w_{0}$, is smaller than the corresponding one of simple holographic dark energy model [13], and it lies in the phantom regime. Furthermore, note that the left and right bounds are larger as expected, since we have the extra freedom in varying the gravitational constant. Finally, $w_{1}$ is slightly larger comparing to simple holographic dark energy scenario.

\section{B. Non-flat universe}

We use the combination of observational data from SNIa, BAO and CMB to construct the likelihood contours for the free model parameters, which in this case are the dimensionless quantities $\Omega_{\Lambda 0}, c, \Delta_{G}$ and $\Omega_{k 0}$, together with $H_{0}$. In Fig. 2 we present the corresponding $1 D$ and $2 D$ likelihood-contours, not only for the parameters used in the fits, but also for the derived ones. Additionally, in Table $\amalg$ we present the $1 \sigma$ best-fit values of the model parameters. 

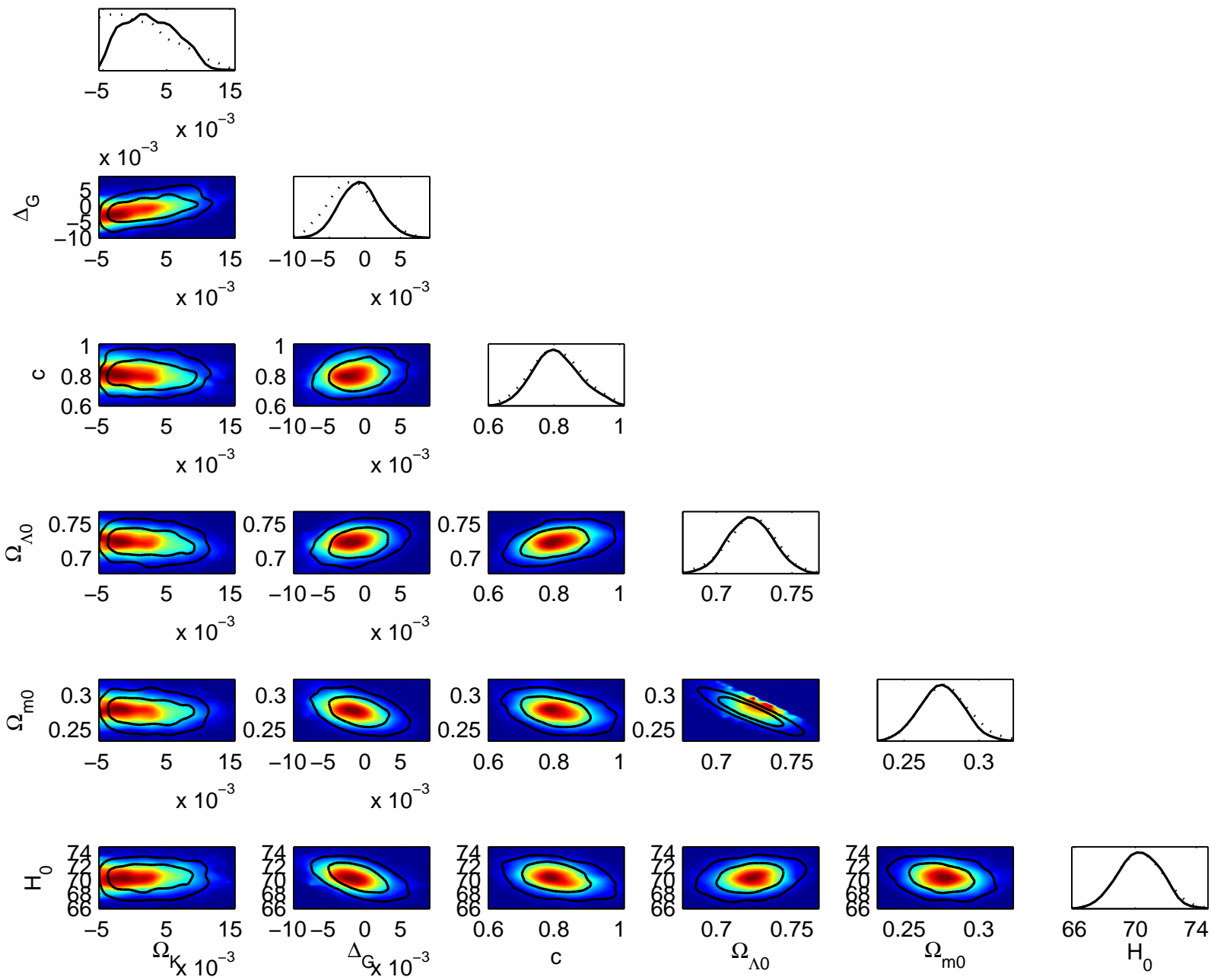

FIG. 2: (Color online) The $1 D$ and $2 D$ likelihood plots, in the non-flat scenario, using SN, BAO, CMB and OHD observational data. The curves stand for the $1 \sigma$ and $2 \sigma$ regions. All quantities are dimensionless, apart from $H_{0}$ which is measured in $\mathrm{km} \mathrm{s}^{-1} \mathrm{Mpc}^{-1}$.

\begin{tabular}{c|c|c|c|c|c}
\hline \hline$\chi_{\min }^{2}$ & $\Omega_{\Lambda 0}(1 \sigma)$ & $c(1 \sigma)$ & $\Delta_{G}(1 \sigma)$ & $\Omega_{k 0}(1 \sigma)$ & $H_{0}(1 \sigma)$ \\
\hline 477.4 & $0.72_{-0.03}^{+0.03}$ & $0.80_{-0.14}^{+0.19}$ & $-0.0025_{-0.0050}^{+0.0080}$ & $-0.0013_{-0.0040}^{+0.0130}$ & $70.4_{-2.9}^{+3.0}$ \\
\hline
\end{tabular}

TABLE II: The minimum value of $\chi^{2}$ and the $1 \sigma$ best-fit values of the model parameters, in the non-flat scenario. All quantities are dimensionless, apart from $H_{0}$ which is measured in $\mathrm{km} \mathrm{s}^{-1} \mathrm{Mpc}^{-1}$.

As we observe, the scenario of holographic dark energy with varying gravitational constant in a non-flat background geometry, is compatible with observations. As expected, the extra freedom in varying the gravitational constant leads to larger bounds of all the parameters, comparing to those of usual holographic dark energy models [15]. However, as we see from the above analysis, the variation range for $\Omega_{k 0}$ is relatively narrow comparing to the physically interesting range arising from the usual investigations of the literature [31]. This feature has to be considered as a disadvantage of the present scenario. 
Since we have approximated the dark energy equation-of-state parameter as $w(z) \approx w_{0}+w_{1}\left(\frac{z}{1+z}\right)$, with $w_{0}$ and $w_{1}$ given by (17), (18), we can easily estimate that in $1 \sigma$ :

$$
\begin{aligned}
& w_{0}=-1.04_{-0.20}^{+0.15} \\
& w_{1}=0.15_{-0.10}^{+0.05}
\end{aligned}
$$

As we see, the present value of $w$, namely $w_{0}$, can lie in the phantom regime. Additionally, note that the best-fit values are very close to those of the flat scenario, since the best-fit value for $\Omega_{k 0}$ is very close to zero. However, the left and right bounds are larger as expected. Finally, note that the best-fit values of both $w_{0}$ and $w_{1}$ are in very good agreement with the corresponding ones of usual (constant- $G$ ) holographic dark energy models [15], but the bounds are larger due to the extra freedom in the $G$-variation.

\section{CONCLUSIONS}

In this work we confronted the scenario of holographic dark energy with varying gravitational constant, with data from SNIa, CMB, BAO and OHD observations. We performed independent fittings for flat and non-flat background geometries, and we extracted the corresponding observational constraints on the free model parameters of each case.

In the case of a flat universe, we deduced that the scenario at hand is compatible with observations, and we presented the corresponding contour-plots on the free parameters, namely $\Omega_{\Lambda 0}, c$ and $\Delta_{G}$, taking into account the uncertainty in $H_{0}$. The gravitational constant variation is inside the corresponding bounds acquired from independent estimations of the literature, and this acts as a self-consistency test of the model. The corresponding $1 \sigma$-bounds are presented in Table I. Moreover, the best-fit value of the present dark energy equation-of-state parameter is smaller than the corresponding one of simple holographic dark energy models [15], lying inside the phantom regime $\left(w_{0}=-1.042_{-0.124}^{+0.131}\right)$. Finally, the left and right bounds are larger as expected, since the scenario at hand possesses the additional freedom of varying the gravitational constant.

For the non-flat geometry, we showed that the scenario at hand is compatible with observations. We constructed the corresponding likelihood contours of the free parameters $\Omega_{\Lambda 0}, \Omega_{k 0}, c$ and $\Delta_{G}$, taking into account the uncertainty in $H_{0}$, and for clarity we presented the $1 \sigma$-bounds in Table II. Furthermore, the best-fit value of the present dark energy equation-of-state parameter lies inside the phantom regime $\left(w_{0}=-1.042_{-0.204}^{+0.153}\right)$, and it is in agreement with the corresponding one of usual holographic dark energy models [15]. However, as expected, the additional variation of the gravitational constant leads to an increase on the extreme values.

In summary, we conclude that the scenario of holographic dark energy with varying gravitational constant can be a candidate for the description of dark energy.

\section{Acknowledgments}

The data fitting is based on the publicly available CosmoMC package a Markov Chain Monte Carlo (MCMC) code. L. Xu is supported by NSF (10703001), SRFDP (20070141034) of P.R. China.

\section{Appendix: Observational data and constraints}

In this appendix we briefly review the main sources of observational constraints used in this work, namely Type Ia Supernovae, Baryon Acoustic Oscillations, Cosmic Microwave Background $\left[l_{A}\left(z_{*}\right), R\left(z_{*}\right), z_{*}\right]$ and Observational Hubble Data (OHD).

\section{Type Ia Supernovae constraints}

We use the 397 SN Ia Constitution dataset, which includes 397 SN Ia [32]. Following [33, 34], one can obtain the corresponding constraints by fitting the distance modulus $\mu(z)$ as

$$
\mu_{t h}(z)=5 \log _{10}\left[D_{L}(z)\right]+\frac{15}{4} \log _{10} \frac{G_{e f f}}{G}+\mu_{0}
$$


where $G$ is the current value of effective Newton's constant $G_{\text {eff }}$. In this expression $D_{L}(z)$ is the Hubble-free luminosity distance $H_{0} d_{L}(z) / c$, with $H_{0}$ the Hubble constant, defined through the re-normalized quantity $h$ as $H_{0}=$ $100 \mathrm{~km} \mathrm{~s}^{-1} \mathrm{Mpc}^{-1}$, and

$$
\begin{aligned}
d_{L}(z) & =\frac{c(1+z)}{\sqrt{\left|\Omega_{k}\right|}} \operatorname{sinn}\left[\sqrt{\left|\Omega_{k}\right|} \int_{0}^{z} \frac{d z^{\prime}}{H\left(z^{\prime}\right)}\right] \\
\mu_{0} & \equiv 42.38-5 \log _{10} h .
\end{aligned}
$$

where $\operatorname{sinnn}\left(\sqrt{\left|\Omega_{k}\right|} x\right)$ respectively denotes $\sin \left(\sqrt{\left|\Omega_{k}\right|} x\right), \sqrt{\left|\Omega_{k}\right|} x, \sinh \left(\sqrt{\left|\Omega_{k}\right|} x\right)$ for $\Omega_{k}<0, \Omega_{k}=0$ and $\Omega_{k}>0$. Additionally, the observed distance moduli $\mu_{o b s}\left(z_{i}\right)$ of SN Ia at $z_{i}$ is

$$
\mu_{o b s}\left(z_{i}\right)=m_{o b s}\left(z_{i}\right)-M
$$

where $M$ is their absolute magnitudes.

For the SN Ia dataset, the best-fit values of the parameters $p_{s}$ can be determined by a likelihood analysis, based on the calculation of

$$
\chi^{2}\left(p_{s}, M^{\prime}\right) \equiv \sum_{S N} \frac{\left\{\mu_{o b s}\left(z_{i}\right)-\mu_{t h}\left(p_{s}, z_{i}\right)\right\}^{2}}{\sigma_{i}^{2}}=\sum_{S N} \frac{\left\{5 \log _{10}\left[D_{L}\left(p_{s}, z_{i}\right)\right]-m_{o b s}\left(z_{i}\right)+M^{\prime}\right\}^{2}}{\sigma_{i}^{2}},
$$

where $M^{\prime} \equiv \mu_{0}+M$ is a nuisance parameter which includes the absolute magnitude and the parameter $h$. The nuisance parameter $M^{\prime}$ can be marginalized over analytically [35] as

$$
\bar{\chi}^{2}\left(p_{s}\right)=-2 \ln \int_{-\infty}^{+\infty} \exp \left[-\frac{1}{2} \chi^{2}\left(p_{s}, M^{\prime}\right)\right] d M^{\prime}
$$

resulting to

$$
\bar{\chi}^{2}=A-\frac{B^{2}}{C}+\ln \left(\frac{C}{2 \pi}\right)
$$

with

$$
\begin{aligned}
A & =\sum_{S N} \frac{\left\{5 \log _{10}\left[D_{L}\left(p_{s}, z_{i}\right)\right]-m_{o b s}\left(z_{i}\right)\right\}^{2}}{\sigma_{i}^{2}} \\
B & =\sum_{S N} \frac{5 \log _{10}\left[D_{L}\left(p_{s}, z_{i}\right)\right]-m_{o b s}\left(z_{i}\right)}{\sigma_{i}^{2}}, \\
C & =\sum_{S N} \frac{1}{\sigma_{i}^{2}} .
\end{aligned}
$$

Relation (A.3) has a minimum at the nuisance parameter value $M^{\prime}=B / C$, which contains information of the values of $h$ and $M$. Therefore, one can extract the values of $h$ and $M$ provided the knowledge of one of them. Finally, note that the expression

$$
\chi_{S N}^{2}\left(p_{s}\right)=A-\left(B^{2} / C\right)
$$

which coincides to (A.4) up to a constant, is often used in the likelihood analysis [33, 35, 36], and thus in this case the results will not be affected by a flat $M^{\prime}$ distribution.

\section{Baryon Acoustic Oscillation constraints}

The Baryon Acoustic Oscillations are detected in the clustering of the combined 2dFGRS and SDSS main galaxy samples, and measure the distance-redshift relation at $z=0.2$. Additionally, Baryon Acoustic Oscillations in the clustering of the SDSS luminous red galaxies measure the distance-redshift relation at $z=0.35$. The observed scale of the BAO calculated from these samples, as well as from the combined sample, are jointly analyzed using estimates of the correlated errors to constrain the form of the distance measure $D_{V}(z)[37-39]$

$$
D_{V}(z)=\left[(1+z)^{2} D_{A}^{2}(z) \frac{c z}{H(z)}\right]^{1 / 3}
$$


In this expression $D_{A}(z)$ is the proper (not comoving) angular diameter distance, which has the following relation with $d_{L}(z)$

$$
D_{A}(z)=\frac{d_{L}(z)}{(1+z)^{2}}
$$

The peak positions of the BAO depend on the ratio of $D_{V}(z)$ to the sound horizon size at the drag epoch (where baryons were released from photons) $z_{d}$, which can be obtained by using a fitting formula [40]:

$$
z_{d}=\frac{1291\left(\Omega_{m} h^{2}\right)^{-0.419}}{1+0.659\left(\Omega_{m} h^{2}\right)^{0.828}}\left[1+b_{1}\left(\Omega_{b} h^{2}\right)^{b_{2}}\right],
$$

with

$$
\begin{aligned}
& b_{1}=0.313\left(\Omega_{m} h^{2}\right)^{-0.419}\left[1+0.607\left(\Omega_{m} h^{2}\right)^{0.674}\right] \\
& b_{2}=0.238\left(\Omega_{m} h^{2}\right)^{0.223} .
\end{aligned}
$$

In this work we use the data of $r_{s}\left(z_{d}\right) / D_{V}(z)$ extracted from the Sloan Digital Sky Survey (SDSS) and the Two Degree Field Galaxy Redshift Survey (2dFGRS) [39], which are listed in Table III] with $r_{s}(z)$ the comoving sound horizon size

$$
r_{s}(z)=c \int_{0}^{t} \frac{c_{s} d t}{a}=c \int_{0}^{a} \frac{c_{s} d a}{a^{2} H}=c \int_{z}^{\infty} d z \frac{c_{s}}{H(z)}=\frac{c}{\sqrt{3}} \int_{0}^{1 /(1+z)} \frac{d a}{a^{2} H(a) \sqrt{1+\left(3 \Omega_{b} /\left(4 \Omega_{\gamma}\right) a\right)}},
$$

where $c_{s}$ is the sound speed of the photon-baryon fluid [41-43]:

$$
c_{s}^{-2}=3+\frac{4}{3} \times \frac{\rho_{b}(z)}{\rho_{\gamma}(z)}=3+\frac{4}{3} \times\left(\frac{\Omega_{b}}{\Omega_{\gamma}}\right) a,
$$

and $\Omega_{\gamma}=2.469 \times 10^{-5} h^{-2}$ for $T_{C M B}=2.75 K$.

\begin{tabular}{c|l}
\hline \hline$z$ & $r_{s}\left(z_{d}\right) / D_{V}(z)$ \\
\hline 0.2 & $0.1905 \pm 0.0061$ \\
\hline 0.35 & $0.1097 \pm 0.0036$ \\
\hline
\end{tabular}

TABLE III: The observational $r_{s}\left(z_{d}\right) / D_{V}(z)$ data [38].

Using the data of BAO in Table [II, the inverse covariance matrix $V^{-1}$ reads [38]:

$$
V^{-1}=\left(\begin{array}{cc}
30124.1 & -17226.9 \\
-17226.9 & 86976.6
\end{array}\right)
$$

Thus, finally, the $\chi_{B A O}^{2}\left(p_{s}\right)$ is given as

$$
\chi_{B A O}^{2}\left(p_{s}\right)=X^{t} V^{-1} X,
$$

where $X$ is a column vector formed from the values of theory minus the corresponding observational data, with

$$
X=\left(\begin{array}{c}
\frac{r_{s}\left(z_{d}\right)}{D_{V}(0.2)}-0.190533 \\
\frac{r_{s}\left(z_{d}\right)}{D_{V}(0.35)}-0.109715
\end{array}\right)
$$

( $X^{t}$ denotes the transpose). 


\section{Cosmic Microwave Background constraints}

The CMB shift parameter $R$ is provided by [44]

$$
R\left(z_{*}\right)=\frac{\sqrt{\Omega_{m} H_{0}^{2}}}{\sqrt{\left|\Omega_{k}\right|}} \operatorname{sinn}\left[\sqrt{\left|\Omega_{k}\right|} \int_{0}^{z_{*}} \frac{d z^{\prime}}{H\left(z^{\prime}\right)}\right],
$$

where the redshift $z_{*}$ (the decoupling epoch of photons) is obtained using the fitting function [45]

$$
z_{*}=1048\left[1+0.00124\left(\Omega_{b} h^{2}\right)^{-0.738}\right]\left[1+g_{1}\left(\Omega_{m} h^{2}\right)^{g_{2}}\right]
$$

and where the functions $g_{1}$ and $g_{2}$ read

$$
\begin{aligned}
& g_{1}=0.0783\left(\Omega_{b} h^{2}\right)^{-0.238}\left[1+39.5\left(\Omega_{b} h^{2}\right)^{0.763}\right]^{-1} \\
& g_{2}=0.560\left[1+21.1\left(\Omega_{b} h^{2}\right)^{1.81}\right]^{-1} .
\end{aligned}
$$

Additionally, the acoustic scale is related to the first distance ratio and is expressed as

$$
l_{A}=\frac{\pi}{r_{s}\left(z_{*}\right)} \frac{c}{\sqrt{\left|\Omega_{k}\right|}} \operatorname{sinn}\left[\sqrt{\left|\Omega_{k}\right|} \int_{0}^{z_{*}} \frac{d z^{\prime}}{H\left(z^{\prime}\right)}\right] .
$$

Using the data of $l_{A}, R, z_{*}$ from [31] which are listed in Table IV] and their covariance matrix of $\left[l_{A}\left(z_{*}\right), R\left(z_{*}\right), z_{*}\right]$ presented in [31]:

$$
C^{-1}=\left(\begin{array}{ccc}
1.800 & 27.968 & -1.103 \\
27.968 & 5667.577 & -92.263 \\
-1.103 & -92.263 & 2.923
\end{array}\right)
$$

we can finally calculate the likelihood $L$ as $\chi_{C M B}^{2}=-2 \ln L$ :

$$
\chi_{C M B}^{2}\left(p_{s}\right)=\triangle d_{i}\left[C^{-1}\left(d_{i}, d_{j}\right)\right]\left[\triangle d_{i}\right]^{t},
$$

where $\triangle d_{i}=d_{i}-d_{i}^{d a t a}$ is a row vector, and $d_{i}=\left(l_{A}, R, z_{*}\right)$.

\begin{tabular}{ccc}
\hline \hline & 5-year maximum likelihood & error, $\sigma$ \\
\hline$l_{A}\left(z_{*}\right)$ & 302.10 & 0.86 \\
$R\left(z_{*}\right)$ & 1.710 & 0.019 \\
$z_{*}$ & 1090.04 & 0.93 \\
\hline \hline
\end{tabular}

TABLE IV: The values of $l_{A}\left(z_{*}\right), R\left(z_{*}\right)$, and $z_{*}$, from 5-year WMAP results [31].

\section{Observational Hubble Data constraints}

The observational Hubble data are based on differential ages of the galaxies [46]. In [47], Jimenez et al. obtained an independent estimate for the Hubble parameter using the method developed in [46], and used it to constrain the equation of state of dark energy. The Hubble parameter, depending on the differential ages as a function of the redshift $z$, can be written as

$$
H(z)=-\frac{1}{1+z} \frac{d z}{d t}
$$

Therefore, once $d z / d t$ is known, $H(z)$ is directly obtained [48]. By using the differential ages of passively-evolving galaxies from the Gemini Deep Deep Survey (GDDS) [49] and archival data [50], Simon et al. obtained $H(z)$ in the range of $0 \lesssim z \lesssim 1.8$ [48]. The twelve observational Hubble data from [51] are listed in TableV] 


\begin{tabular}{c|lllllllllllll}
\hline \hline$z$ & 0 & 0.1 & 0.17 & 0.27 & 0.4 & 0.48 & 0.88 & 0.9 & 1.30 & 1.43 & 1.53 & 1.75 \\
\hline$H(z)\left(\mathrm{km} \mathrm{s}^{-1} \mathrm{Mpc}^{-1}\right)$ & 74.2 & 69 & 83 & 77 & 95 & 97 & 90 & 117 & 168 & 177 & 140 & 202 \\
\hline $1 \sigma$ uncertainty & \pm 3.6 & \pm 12 & \pm 8 & \pm 14 & \pm 17 & \pm 60 & \pm 40 & \pm 23 & \pm 17 & \pm 18 & \pm 14 & \pm 40 \\
\hline
\end{tabular}

TABLE V: The observational $H(z)$ data [51].

Furthermore, in [52] the authors used the BAO scale as a standard ruler in the radial direction, and they obtained three more additional data: $H(z=0.24)=79.69 \pm 2.32, H(z=0.34)=83.8 \pm 2.96$, and $H(z=0.43)=86.45 \pm 3.27$.

The best-fit values of the model parameters from observational Hubble data [48] are determined by minimizing

$$
\chi_{H u b}^{2}\left(p_{s}\right)=\sum_{i=1}^{15} \frac{\left[H_{t h}\left(p_{s} ; z_{i}\right)-H_{o b s}\left(z_{i}\right)\right]^{2}}{\sigma^{2}\left(z_{i}\right)}
$$

where $p_{s}$ denotes the parameters contained in the model, $H_{t h}$ is the predicted value for the Hubble parameter, $H_{o b s}$ is the observed value, $\sigma\left(z_{i}\right)$ is the standard deviation measurement uncertainty, and the summation runs over the 15 observational Hubble data points at redshifts $z_{i}$.

[1] S. Perlmutter et al. [Supernova Cosmology Project Collaboration], Astrophys. J. 517, 565 (1999).

[2] C. L. Bennett et al., Astrophys. J. Suppl. 148, 1 (2003).

[3] M. Tegmark et al. [SDSS Collaboration], Phys. Rev. D 69, 103501 (2004).

[4] S. W. Allen, et al., Mon. Not. Roy. Astron. Soc. 353, 457 (2004).

[5] O. Bertolami, Nuovo Cim. B 93 (1986) 36; J. Sola and H. Stefancic, Phys. Lett. B 624, 147 (2005); J. Sola and H. Stefancic, Mod. Phys. Lett. A 21, 479 (2006); I. L. Shapiro and J. Sola, Phys. Lett. B 682, 105 (2009).

[6] B. Ratra and P. J. E. Peebles, Phys. Rev. D 37, 3406 (1988); C. Wetterich, Nucl. Phys. B 302, 668 (1988); A. R. Liddle and R. J. Scherrer, Phys. Rev. D 59, 023509 (1999); I. Zlatev, L. M. Wang and P. J. Steinhardt, Phys. Rev. Lett. 82, 896 (1999); Z. K. Guo, N. Ohta and Y. Z. Zhang, Mod. Phys. Lett. A 22, 883 (2007); S. Dutta, E. N. Saridakis and R. J. Scherrer, Phys. Rev. D 79, 103005 (2009).

[7] R. R. Caldwell, Phys. Lett. B 545, 23 (2002); R. R. Caldwell, M. Kamionkowski and N. N. Weinberg, Phys. Rev. Lett. 91, 071301 (2003); S. Nojiri and S. D. Odintsov, Phys. Lett. B 562, 147 (2003); V. K. Onemli and R. P. Woodard, Phys. Rev. D 70, 107301 (2004); M. R. Setare, J. Sadeghi, A. R. Amani, Phys. Lett. B 666, 288, (2008); X. m. Chen, Y. g. Gong and E. N. Saridakis, JCAP 0904, 001 (2009); E. N. Saridakis, Nucl. Phys. B 819, 116 (2009).

[8] B. Feng, X. L. Wang and X. M. Zhang, Phys. Lett. B 607, 35 (2005); Z. K. Guo, et al., Phys. Lett. B 608, 177 (2005); M.-Z Li, B. Feng, X.-M Zhang, JCAP, 0512, 002 (2005); B. Feng, M. Li, Y.-S. Piao and X. Zhang, Phys. Lett. B 634, 101 (2006); M. R. Setare, Phys. Lett. B 641, 130 (2006); W. Zhao and Y. Zhang, Phys. Rev. D 73, 123509 (2006); M. R. Setare, J. Sadeghi, and A. R. Amani, Phys. Lett. B 660, 299 (2008); M. R. Setare and E. N. Saridakis, Phys. Lett. B 668, 177 (2008); M. R. Setare and E. N. Saridakis, JCAP 0809, 026 (2008); M. R. Setare and E. N. Saridakis, Int. J. Mod. Phys. D 18, 549 (2009).

[9] S. Nojiri and S. D. Odintsov, Int. J. Geom. Meth. Mod. Phys. 4, 115 (2007); S. Nojiri and S. D. Odintsov, arXiv:0801.4843 [astro-ph]; T. P. Sotiriou and V. Faraoni, arXiv:0805.1726 [gr-qc].

[10] L. Perivolaropoulos, JCAP 0510, 001 (2005).

[11] I. Y. Aref'eva and A. S. Koshelev, JHEP 0702, 041 (2007); L. P. Chimento, R. Lazkoz, R. Maartens and I. Quiros, JCAP 0609, 004 (2006); J. Sadeghi, M. R. Setare, A. Banijamali and F. Milani, Phys. Lett. B 662, 92 (2008).

[12] S. D. H. Hsu, Phys. Lett. B 594, 13 (2004).

[13] M. Li, Phys. Lett. B 603, 1 (2004).

[14] A. G. Cohen, D. B. Kaplan and A. E. Nelson, Phys. Rev. Lett. 82, 4971 (1999); P. Horava and D. Minic, Phys. Rev. Lett. 85, 1610 (2000); S. D. Thomas, Phys. Rev. Lett. 89, 081301 (2002).

[15] Q. G. Huang and Y. G. Gong, JCAP 0408, 006 (2004); J. y. Shen, B. Wang, E. Abdalla and R. K. Su, Phys. Lett. B 609, 200 (2005); H. C. Kao, W. L. Lee and F. L. Lin Phys. Rev. D 71123518 (2005); X. Zhang and F. Q. Wu, Phys. Rev. D 72, 043524 (2005); B. Wang, C. Y. Lin and E. Abdalla, Phys. Lett. B 637, 357 (2006); Z. Chang, F. Q. Wu and X. Zhang, Phys. Lett. B 633, 14 (2006); C. Feng, B. Wang, Y. Gong and R. K. Su, JCAP 0709, 005 (2007); L. Xu, J. Lu and W. Li, arXiv:0905.4174 [astro-ph.CO].

[16] Q. G. Huang and M. Li, JCAP 0408, 013 (2004).

[17] K. Enqvist and M. S. Sloth, Phys. Rev. Lett. 93, 221302 (2004); M. Ito, Europhys. Lett. 71, 712 (2005); Q. G. Huang and M. Li, JCAP 0503, 001 (2005); D. Pavon and W. Zimdahl, Phys. Lett. B 628, 206 (2005); B. Wang, Y. Gong and E. Abdalla, Phys. Lett. B 624, 141 (2005); H. Kim, H. W. Lee and Y. S. Myung, Phys. Lett. B 632, 605 (2006); S. Nojiri and S. D. Odintsov, Gen. Rel. Grav. 38, 1285 (2006); E. Elizalde, S. Nojiri, S. D. Odintsov and P. Wang, Phys. Rev. D 71, 103504 (2005); B. Hu and Y. Ling, Phys. Rev. D 73, 123510 (2006); H. Li, Z. K. Guo and Y. Z. Zhang, Int. J. Mod. Phys. 
D 15, 869 (2006); M. R. Setare, Phys. Lett. B 642, 1 (2006); M. R. Setare, Phys. Lett. B642, 421, (2006); E. N. Saridakis, Phys. Lett. B 660, 138 (2008); E. N. Saridakis, JCAP 0804, 020 (2008); E. N. Saridakis, Phys. Lett. B 661, 335 (2008).

[18] L. Amendola, Phys. Rev. D 62, 043511 (2000); D. Comelli, M. Pietroni and A. Riotto, Phys. Lett. B 571, 115 (2003); M. R. Setare, JCAP 0701, 023 (2007); M. R. Setare and E. N. Saridakis, Phys. Lett. B 670, 1 (2008).

[19] S. D'Innocenti, G. Fiorentini, G. G. Raffelt, B. Ricci and A. Weiss, Astron. Astrophys. 312, 345 (1996); K. Umezu, K. Ichiki and M. Yahiro, Phys. Rev. D 72, 044010 (2005); S. Nesseris and L. Perivolaropoulos, Phys. Rev. D 73, 103511 (2006); J. P. W. Verbiest et al., arXiv:astro-ph/0801.2589.

[20] G. S. Bisnovatyi-Kogan, Int. J. Mod. Phys. D 15, 1047 (2006).

[21] Damour T., et al, Phys. Rev. Lett. 61, 1151 (1988).

[22] D.B. Guenther, Phys. Lett. B 498, 871 (1998).

[23] S. Ray and U. Mukhopadhyay, Int. J. Mod. Phys. D 16, 1791 (2007).

[24] M. Jamil, E. N. Saridakis and M. R. Setare, Phys. Lett. B 679, 172 (2009).

[25] B. Guberina, R. Horvat and H. Nikolic, Phys. Rev. D 72, 125011 (2005).

[26] M. Chevallier and D. Polarski, Int. J. Mod. Phys. D 10, 213 (2001).

[27] A. Lewis and S. Bridle, Phys. Rev. D 66103511 (2002).

[28] E. Gaztanaga, E. Garcia-Berro, J. Isern, E. Bravo and I. Dominguez, Phys. Rev. D 65, 023506 (2002).

[29] M. Biesiada and B. Malec, Mon. Not. R. Astron. Soc. 350, 644 (2004).

[30] Q. J. Zhang and Y. L. Wu, arXiv:0905.1234 [astro-ph.CO].

[31] E. Komatsu et al., [WMAP Collaboration], Astrophys. J. Suppl. 180330 (2009).

[32] M. Hicken et al., Astrophys. J. 700, 1097 (2009).

[33] E. Garcia-Berro, E. Gaztanaga, J. Isern, O. Benvenuto and L. Althaus, arXiv:astro-ph/9907440; A. Riazuelo, J. Uzan, Phys.Rev. D 66023525 (2002); V. Acquaviva, L. Verde, JCAP 0712001 (2007).

[34] R.Gannouji, D. Polarski, JCAP 0805, 018 (2008).

[35] S. Nesseris and L. Perivolaropoulos, Phys. Rev. D 72, 123519 (2005); L. Perivolaropoulos, Phys. Rev. D 71, 063503 (2005); E. Di Pietro and J. F. Claeskens, Mon. Not. Roy. Astron. Soc. 341, 1299 (2003); A. C. C. Guimaraes, J. V. Cunha and J. A. S. Lima, JCAP 0910, 010 (2009).

[36] L. Xu, W. Li, J. Lu, JCAP 0907, 031 (2009).

[37] D. J. Eisenstein et al., Astrophys. J. 633560 (2005).

[38] W.J. Percival et al., arXiv:0907.1660 [astro-ph.CO].

[39] W. J. Percival et al., Mon. Not. Roy. Astron. Soc. 3811053 (2007).

[40] D. J. Eisenstein and W. Hu, Astrophys. J. 496605 (1998).

[41] W. Hu and N. Sugiyama, Astrophys. J. 444489 (1995).

[42] W. Hu, M. Fukugita, M. Zaldarriaga and M. Tegmark, Astrophys. J. 549669 (2001).

[43] R. R. Caldwell and M. Doran, Phys. Rev. D 69103517 (2004).

[44] J. R. Bond, G. Efstathiou and M. Tegmark, Mon. Not. Roy. Astron. Soc. 291 L33 (1997).

[45] W. Hu and N. Sugiyama, Astrophys. J. 471542 (1996).

[46] R. Jimenez and A. Loeb, Astrophys. J. 57337 (2002).

[47] R. Jimenez, L. Verde, T. Treu and D. Stern, Astrophys. J. 593622 (2003).

[48] J. Simon, L. Verde and R. Jimenez, Phys. Rev. D 71123001 (2005).

[49] R. G. Abraham et al., Astron. J. 1272455 (2004).

[50] J. Dunlop et al., Nature 381581 (1996); H. Spinrad et al., Astrophys. J. 484581 (1997); T. Treu et al., Mon. Not. Roy. Astron. Soc. 3081037 (1999); T. Treu et al., Mon. Not. Roy. Astron. Soc. 326221 (2001); T. Treu et al., Astrophys. J. Lett. 564 L13 (2002); L. A. Nolan, J. S. Dunlop, R. Jimenez and A. F. Heavens, Mon. Not. Roy. Astron. Soc. 341464 (2003).

[51] A. G. Riess et al., Astrophys. J. 699, 539 (2009); D. Stern et al., JCAP 1002, 008 (2010).

[52] E. Gaztanaga, A. Cabre and L. Hui, arXiv:0807.3551 [astro-ph]. 